\title{
Quantitative SEM-EDS Analysis of Semi-transparent Samples
}

\author{
Stephen Boona
}

\section{The Ohio State University, Columbus, Ohio, United States}

Here we report on empirical guidelines for quantitative energy dispersive- $\mathrm{X}$-ray spectroscopy (EDS) analysis of sub-micron specimens at electron beam energies of $30 \mathrm{keV}$ and below. The impetus for this study emerges from the fact that imaging and analysis techniques involving transmission of an electron beam through thin samples have traditionally been applied only within instruments designed specifically for this purpose. Such Transmission Electron Microscopes (TEMs) typically operate at accelerating voltages in the range of $100 \mathrm{kV}$ to $300 \mathrm{kV}$, which are high enough that minimal absorption of the beam occurs within the sample. While TEMs have been wildly successful in extracting an enormous variety of information from an enormous variety of materials, recent years have seen an increased crossover of techniques originally developed for TEMs being utilized as well in Scanning Electron Microscopes (SEMs), wherein the maximum available accelerating voltage is typically only $30 \mathrm{kV}$. Examples of transmission-based techniques successfully applied in SEMs include STEM imaging, Low Energy Nano Diffraction [1], Transmission Kikuchi Diffraction [2], and Electron Energy Loss Spectroscopy [3].

The order of magnitude difference in accelerating voltages available in SEMs and TEMs means samples that are nominally transparent in a TEM may be only semi-transparent in an SEM, i.e., some or most of the beam is transmitted, but beam-sample interactions exceed what can be captured by simple perturbative treatments. This means one must carefully consider which assumptions about electron and photon absorption are still appropriate when accounting for the difference in beam-sample interactions at these lower energies, especially in the context of quantitative composition analysis via EDS. Although robust models exist for accurate EDS analysis of bulk SEM specimens up to $30 \mathrm{kV}$, as well as thin TEM samples down to $100 \mathrm{kV}$, there exists sparse guidance on how to accurately analyze EDS data from thin TEM specimens at SEM voltages. Since the landing energy of electron beams can be set arbitrarily low in SEMs, and every element in the periodic table has at least one characteristic $\mathrm{x}$-ray transition below $5 \mathrm{keV}$, it is not necessarily clear a priori if superior quantification results can be obtained by using low energy beams to avoid transmission, or if better results come from using the more easily distinguishable x-ray transitions at energies above $10 \mathrm{keV}$ despite the likelihood of incomplete beam absorption at such energies.

This study provides insight into this question by comparing the results of EDS analyses of thin samples made from bulk pieces of binary compounds with well-defined atomic ratios: high purity $\mathrm{Al}_{2} \mathrm{O}_{3}$ ( $\mathrm{Kyocera}$ Global) and high purity $\mathrm{FeS}_{2}$ (Alfa Aesar). Small pieces of each material were loaded into an FEI Nova DualBeam Focused Ion Beam (FIB) instrument to extract lamellae for analysis. The lamellae were thinned in the FIB to create within each sample multiple terraced segments with thicknesses varying between approximately 0.4 and 1.8 microns. Figure 1(a) shows a backscatter electron image of the top-down view of the protective Pt layer atop the $\mathrm{FeS}_{2}$ lamella after thinning. Top-down images like this were used to determine the approximate width of each segment within the lamellae.

The thinned samples were then loaded into a ThermoFisher Apreo LoVac Schottky Field Emission SEM for EDS analysis. The accelerating voltage was varied in steps from $3 \mathrm{kV}$ to $30 \mathrm{kV}$ while EDS linescan data were collected using an EDAX Octane Elect EDS SDD via the EDAX TEAM software package. The electron beam current and $\mathrm{x}$-ray detector amp time were varied to keep the count rate and dead time approximately constant. 
An example of the observed thickness-dependent variation in detected x-ray intensity is provided in Figure 1(b), which shows the normalized intensity of the $\mathrm{S}$ K-edge for different accelerating voltages. Deviations of the normalized intensity from unity were used to determine if any beam transmission occurred within a given segment. The linescan data were analyzed in the EDAX TEAM software to convert raw peak intensities into $\mathrm{Al} / \mathrm{O}$ and $\mathrm{Fe} / \mathrm{S}$ atomic ratios. This analysis was performed using different models available within TEAM, including the standard "eZAF" model for bulk specimens, as well as the "MThin" approach intended for transparent materials, the latter based on the well-known Cliff-Lorimer (CL) k-factor method of comparing intensity ratios. The optional absorption correction was added within MThin and the theoretical density of each material was also specified. The modeled sample thickness was iteratively set to values of $500 \mathrm{~nm}, 750 \mathrm{~nm}$, and $1000 \mathrm{~nm}$, and the resulting composition for each segment was calculated. A comparison is provided in Table 1 of conditions in which the software produced 'correct' results, defined as within $2 \%$ of the expected value for each material.

The most obvious trend among the data in Table 1 is that there are no instances in which a correct result is obtained at $10 \mathrm{keV}$ or below, nor with the eZAF bulk absorption model. Instead, we see accurate composition analysis only when we consider the signals generated by higher energy, partially transmitted beams after running them through the MThin model. These results can be interpreted by considering the tradeoffs between factors such as the ease of uniquely identifying high vs low energy peaks, the magnitude of overvoltage, the degree of transmission, and the role of energy-dependent x-ray mass absorption coefficients.

More broadly, the results of this study suggest the best approach to quantitative SEM-EDS analysis of semi-transparent specimens is to deal with transmission not by avoiding it with low accelerating voltages, but to emphasize it by using beam energies high enough to ensure some transmission, then account for the semi-transparency with conventional CL-type quantification models that consider the sample thickness and density [4].
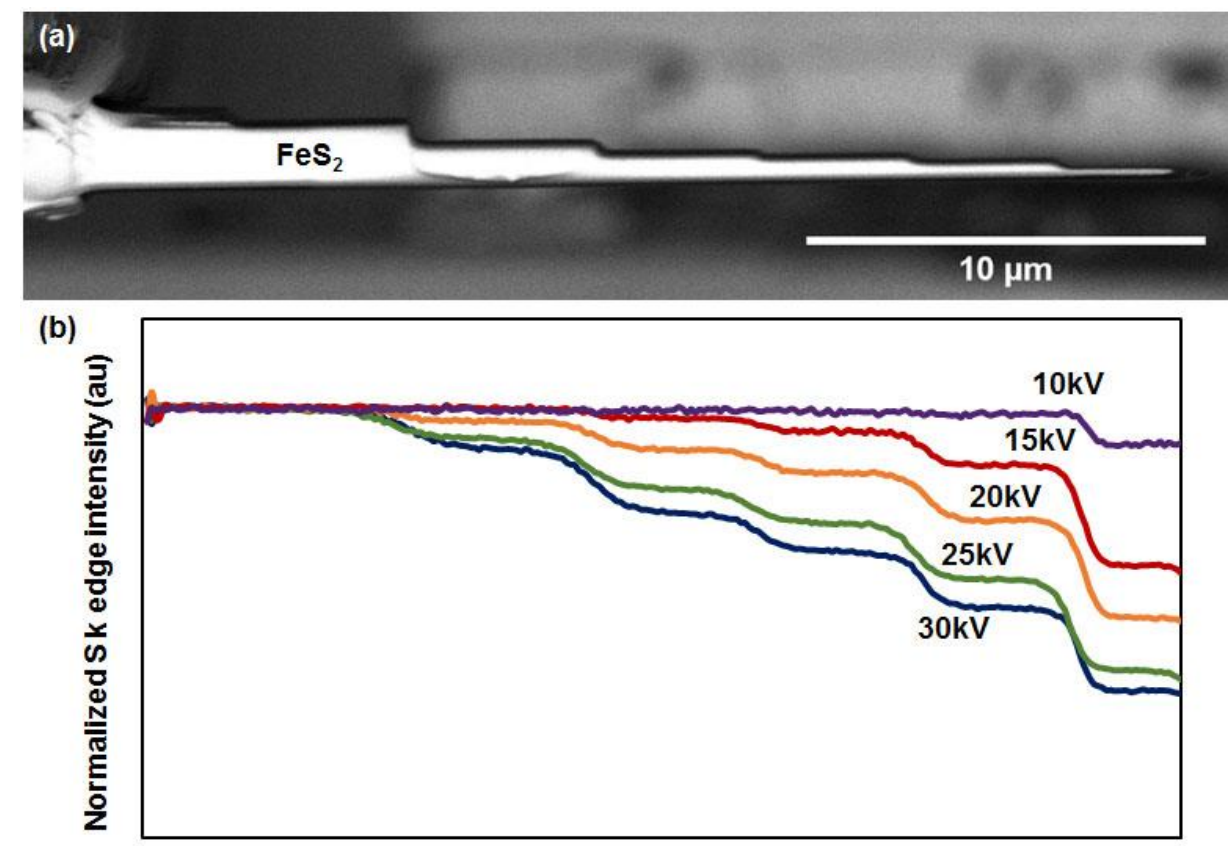

Scan position $(\mu \mathrm{m})$ 
Figure 1. (a) Backscatter electron image of the protective Pt layer atop the FeS2 lamella prepared via FIB and thinned to create six segments of varying widths. (b) Corresponding EDS linescan data showing the normalized intensity of the S K-edge data collected from each segment, with incident accelerating voltages as labeled.

\begin{tabular}{|cccc|}
\hline Material & Estimated segment thickness $(\mathbf{n m})$ & Beam Energy (keV) & Model \\
\hline $\mathrm{Al}_{2} \mathrm{O}_{3}$ & 1169 & 20 & MThin $1000 \mathrm{~nm}$ \\
\hline $\mathrm{Al}_{2} \mathrm{O}_{3}$ & 885 & 30 & MThin $1000 \mathrm{~nm}$ \\
\hline $\mathrm{Al}_{2} \mathrm{O}_{3}$ & 885 & 20 & MThin $750 \mathrm{~nm}$ \\
\hline $\mathrm{Al}_{2} \mathrm{O}_{3}$ & 885 & 15 & MThin $750 \mathrm{~nm}$ \\
\hline $\mathrm{Al}_{2} \mathrm{O}_{3}$ & 505 & 15 & MThin $500 \mathrm{~nm}$ \\
\hline $\mathrm{FeS}_{2}$ & 1826 & 30 & MThin 750nm \\
\hline $\mathrm{FeS}_{2}$ & $457,623,809,893,1204,1826$ & 25 & MThin 500nm \\
\hline
\end{tabular}

Figure 2. Table 1. List of conditions under which quantitative analysis of EDS linescan data were accurate within $2 \%$ of the known composition.

References

[1] P. Schweizer et al., Microsc. Microanal. 25 (Suppl 2), p450 (2019)

[2] R.R. Keller and R.H. Geiss, J. Microsc. 245 3, p245-251 (2012)

[3] Y. Yamazawa et al., Microsc. Microanal. 22 (Suppl 3), p50 (2016)

[4] The author gratefully acknowledges discussions with H. Colijn, D. Huber, R. Williams, and D. Veghte, as well as direct support from Ohio State's Center for Electron Microscopy and Analysis (CEMAS). 